\title{
0 uso de álcool por adolescentes: Uma comparação por gênero
}

\author{
Use of alcohol by adolescents: A comparison per gender
}

\author{
Edilane da Silva Freitas ${ }^{[a]}$, Karla Carolina Silveira Ribeiro ${ }^{[b]}$, Ana Alayde Werba Saldanha ${ }^{[c]}$
}

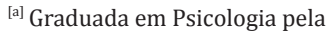
Universidade Federal da Paraíba (UFPB), João Pessoa, PB - Brasil.

${ }^{[\mathrm{b}]}$ Mestre em Psicologia Social, Doutoranda em Psicologia Social pela Universidade Federal da Paraíba (UFPB/UFRN), João Pessoa, PB - Brasil.

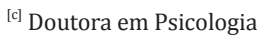
Social Universidade de São Paulo, professora adjunta da Universidade Federal da Paraíba, (UFPB) João Pessoa, PB - Brasil, e-mail: edilane_sf@hotmail.com
}

Recebido: 29/01/2010 Received: 01/29/2010

Aprovado: 23/11/2010 Approved: 11/23/2010

\section{Resumo}

Objetivo: Examinar a relação existente entre o gênero e o uso de álcool em adolescentes. Método: A amostra foi composta por 1.138 adolescentes, dos quais 58\% eram do sexo feminino, com média de idade de 16 anos (DP = 1,45). Para a coleta dos dados foi utilizado um questionário autoaplicável, cujas análises foram feitas por meio de estatística descritiva e teste bivariado, a fim de verificar a existência de associações entre as variáveis. Resultados e discussão: $76 \%$ dos adolescentes afirmam ter ingerido bebida alcoólica pelo menos uma vez na vida, com idade de experimentação aos 14 anos em média. Foi verificada uma associação entre o uso do álcool e religião/religiosidade, trabalho remunerado e satisfação com a vida. Na análise por gênero, observaram-se diferenças entre as variáveis uso de álcool na vida, idade de experimentação, frequência do beber no último mês, motivação para beber e idade de iniciação ao consumo. Os adolescentes identificaram como consequência do uso do álcool: comportamentos sexuais de risco, de violência e ações que não teriam tomado em abstinência. Considerações finais: Os resultados apontam para o estabelecimento de padrões de consumo de álcool maior para mulheres do que para homens em gerações futuras, o que pode estar associado à emancipação social desse grupo populacional.

Palavras-chave: Uso de álcool. Adolescentes. Gênero. 
appoint to the establishment of greater alcohol consumption patterns for women than for men in future generations, which may be associated with social emancipation of the gender group.

Keywords: Use of alcohol. Adolescents. Gender.

\section{Introdução}

As noções de masculino e feminino transcendem a questão das diferenças anatômicas existentes entre homens e mulheres. 0 conceito de gênero denota os significados, atributos, funções e relações socialmente construídas atribuídos a essas distinções de caráter biológico, utilizados, muitas vezes, para legitimar diferentes relações de poder e de opressão (Korin, 2001). Neste paradigma, a figura masculina é tida como ativa, forte, capaz do trabalho físico árduo, produtiva, competitiva e orientada ao mundo externo, confiante, destemida, determinada, realizadora, independente, objetiva, pragmática, racional, emocionalmente equilibrada, profissionalmente competente, financeiramente bem-sucedida e sexualmente impositiva (Wang, Jablonski \& Magalhães, 2006). A mulher, em contrapartida, seria dotada de características opostas, como passividade, debilidade, enfermidade, dependência e sensibilidade (Korin, 2001). As estereotipias que resultam dessa hegemonia masculina desencadeiam comportamentos de saúde prejudiciais, apresentando-se como fator de vulnerabilidade (Grimberg, 1999).

A aprendizagem desses padrões de gênero começa já na infância, nos seus ambientes de convivência, especialmente na família e na vizinhança (Duque-Arrazola, 1997). É no cotidiano da família e nas relações intrafamiliares, já completamente enredados pela hierarquia de gênero, que meninos e meninas vão aprender a forma de agir própria de seu gênero, aceita pelo grupo social em que se encontra inserida. Assim, todas as suas atividades - rotina, brincadeiras, jogos, perspectivas e planos para o futuro - acabam trazendo uma pesada carga de gênero e reproduzindo os papéis masculino e feminino, tais como são postos pela sociedade (TraversoYépez \& Pinheiro, 2005). Além disso, a forma como os pais educam os filhos e as filhas diferem consideravelmente (Larossa, 1989).

O mundo contemporâneo, com suas transformações e revoluções tecnológicas tem impactado bastante na estrutura das famílias e nos papéis de gênero. A inserção da mulher no mercado de trabalho e no cenário social de forma geral, associada às atuais dificuldades econômicas e às reivindicações dos movimentos de mulheres vem fazendo com que a família sofra modificações na sua dinâmica (Hennigen \& Guareschi, 2002). A indústria das substâncias psicoativas acompanha de perto as transformações da sociedade, buscando oportunidades de efetivar a difusão da oferta e a sedução ao consumo (Costa-e-Silva \& Koifman, 1998).

Em estudo com propagandas de bebidas alcoólicas, Pinsky e Silva (1999) perceberam veiculação em uma frequência superior se comparadas aos outros produtos e que três dentre as cinco temáticas trazidas mais comumente por elas eram diretamente relacionáveis às expectativas dos jovens (camaradagem, humor e relaxamento). No Brasil, apesar de existirem leis que proíbem a venda de álcool para adolescentes, elas não vêm sendo cumpridas e, além disso, tem havido, assim como em outros países, estímulo ao consumo por parte de propagandas (Amaral, 2008; Carlini, Carlini-Cotrim, Silva-Filho \& Barbosa, 1990). Portanto faz-se necessário atentar para as populações consumidoras em potencial dessas substâncias, bem como para aspectos imprescindíveis ou relacionados à mudança de comportamento, no sentido de se subsidiar políticas preventivas e ações de enfrentamento aos problemas decorrentes do uso de drogas (El-Guebaly, 2006).

A adolescência, mais do que todas as outras etapas da vida, é marcada pela transitoriedade, em que há uma busca pela construção de uma identidade própria, processo no qual estão implicados os valores tanto dos pais e da cultura de origem quanto também do seu ambiente imediato (Michaud, 2001). É ainda fundamental nessa fase a procura por novas experiências, as trocas e a influência do grupo de amigos, sendo o uso de drogas incluído como fator de socialização e expressão, mas que, se utilizado de forma desmedida, pode causar problemas e afetar toda a sua vida (Schenker \& Minayo, 
2003). O uso de substâncias, segundo Pechansky (2001), é um dos muitos comportamentos "adultos" incorporados pelos jovens, que percebem muito frequentemente a substância como uma expressão de independência e autonomia. Em busca dessa sensação de maturidade, o adolescente se utiliza de uma escala crescente de experimentação com substâncias, começando pelas usadas legalmente pelos adultos, como cigarro e, sobretudo, o álcool; e passando, posteriormente para outras, de caráter ilegal (como maconha e lança perfume, por exemplo). Com a experimentação dessas últimas ou o abuso de substâncias legais (uma intoxicação alcoólica), há um reforço tanto direcionado ao comportamento normal (evitação de uso abusivo) quanto, para alguns indivíduos, ao comportamento desviante da norma social (abuso/dependência de drogas).

Paralelamente às especificidades de cada sujeito, existe um conjunto de crenças e expectativas de efeitos do uso do álcool, que refletem essa história. Mesmo antes do primeiro contato com o álcool, elas já existem, sendo aprendidas vicariamente e influenciam a decisão de beber. A experiência com o álcool pode confirmar expectativas de confiança e bem-estar, fortalecendo as expectativas pré-existentes e especificando-as (Araújo \& Gomes, 1998).

Estudos realizados atestam maior consumo de tabaco, álcool e outras drogas pelos homens (Carlini, Galduróz, Noto \& Nappo, 2002); no entanto essa informação pode estar mudando dadas as transformações que estão acontecendo atualmente no campo da saúde (Kroeff et al., 2004). Durante muito tempo, o consumo do álcool foi associado ao gênero masculino, dadas as baixas estatísticas de mulheres nos serviços de saúde especializados (Cardim \& Azevedo, 1995). Esses dados levantaram discussões que iam tanto na direção do entendimento do alcoolismo como um problema predominantemente masculino (Keller, 1980), como na investigação na perspectiva do comportamento social diante do alcoolismo em mulheres. De acordo com Robins (1993), essa identificação entre álcool e população masculina teria um caráter mais social e as mulheres não estariam nesses serviços graças a uma moral implícita no que se refere ao consumo do álcool e estabelecia padrões mais rígidos para as mulheres, desfavorecendo o consumo na população feminina e estigmatizando aquelas que bebiam.

A partir das conquistas femininas nas últimas décadas, conforme destacado por Souza, Areco e
Silveira Filho (2005), as adolescentes passaram a ter mais liberdade de frequentar locais onde se consomem bebidas alcoólicas, antes restritas aos homens, comportamentos esses que se acompanham do aumento da prevalência de doenças, anteriormente associadas ao sexo masculino. Neste sentido, observa-se a importância de se considerar o espaço social das mulheres e as diferenças de gênero no alcoolismo, as quais modulam o comportamento de beber de homens e mulheres, sendo o conhecimento de tais características fundamentais para a eficácia das ações em saúde.

A partir destas premissas, este estudo teve como objetivo traçar um perfil dos adolescentes com relação ao uso do álcool, associando as características desse consumo com o gênero.

\section{Método}

\section{Característica do estudo}

Trata-se de estudo com caráter descritivo e transversal, tendo como lócus escolas de grande e pequeno porte da rede estadual de ensino, localizadas em diferentes zonas da cidade de João Pessoa, Paraíba.

\section{Participantes}

Participaram deste estudo, 1138 estudantes da rede estadual de ensino da cidade de João Pessoa $\mathrm{PB}$, dos quais $42 \%$ são do sexo masculino e $58 \%$ do sexo feminino. A média de idade dos adolescentes foi de 16 anos (DP = 1,45), variando de 12 a 20, sendo que $9 \%$ encontram-se na faixa etária de 12 a 14 anos, 48\% têm entre 15 e 16 anos, 35\% situam-se na faixa etária dos 17 aos 18 anos, e 8\%, dos 19 aos 20 anos.

Instrumento

Foi utilizado um questionário autoaplicável contendo questões biodemográficas e relacionadas ao uso do álcool (uso na vida, idade da primeira experimentação, frequência de uso nos últimos 30 dias, motivação para o uso e consequências da ingestão), construído com base nos estudos de "The Behavioral Risk Factor Surveillance System" (BRFSS) (USDHHS, 1999); Farias Jr. (2002); De Bem (2003) e Amaral (2008). 


\section{Procedimentos}

A pesquisa foi aprovada pelo Comitê de Ética em pesquisa do Centro de Ciências da Saúde da Universidade Federal da Paraíba e autorizada pela Secretaria Estadual de Educação, procurando respeitar todos os aspectos éticos que trata da pesquisa com seres humanos. A aplicação do questionário foi realizada por pesquisadores devidamente treinados e se deu de forma coletiva em ambiente de sala de aula, após a explicitação dos objetivos, obtenção do consentimento livre e esclarecido dos alunos/as, os esclarecimentos necessários e instruções pertinentes.

\section{Análise de dados}

As análises foram feitas a partir do software SPSS for Windows, mediante estatística descritiva (Medidas de Posição e Variabilidade), estatística NãoParamétrica (Qui-Quadrado) e testes bivariados (teste $T$ de Student) a fim de verificar a existência ou não de associações entre as variáveis do estudo.

\section{Resultados e discussão}

\section{Prevalência e iniciação}

Foi encontrado um índice de uso de álcool na vida de $76 \%$, valor consideravelmente superior ao obtido pelo Centro Brasileiro de Informações sobre Drogas Psicotrópicas, em estudo de abrangência nacional, que foi de 65,2\% (Galduróz, 2004). Dos adolescentes que afirmaram já ter ingerido bebida alcoólica, 41\% declarou ter bebido nos 30 dias que antecederam a realização da pesquisa, com frequência de 1 a 5 vezes para $31 \%$ e de 6 ou mais vezes para $10 \%$. Esse dado fornece indicativos de que grande parte dos jovens não fica apenas na experimentação, mas desenvolve um padrão de uso regular e, para uma parcela considerável deles, também frequente da substância em questão.

A idade da primeira experimentação de bebida alcoólica foi de 14 anos em média (DP = 2,04), dado em conformidade com os resultados do I Levantamento Nacional sobre o uso de álcool (2007) para os adolescentes na faixa etária de 14 a 17 anos - compatível com a idade da maior parte da amostra deste estudo. 0 perfil do uso de álcool pelos adolescentes pode ser observado na Tabela 1.
Tabela 1 - Características do Uso de álcool

\begin{tabular}{|c|c|c|}
\hline Variáveis & Feminino & Masculino \\
\hline Uso na vida & $56 \%$ & $44 \%$ \\
\hline Uso nos últimos 30 dias & $52 \%$ & $48 \%$ \\
\hline $\begin{array}{l}\text { Frequência } \\
1-5 \text { vezes } \\
\geq 6 \text { vezes }\end{array}$ & $\begin{array}{l}52 \% \\
48 \%\end{array}$ & $\begin{array}{l}48 \% \\
58 \%\end{array}$ \\
\hline Idade Experimentação (média) & 14,4 anos & 13,5 anos \\
\hline
\end{tabular}

Fonte: Dados da pesquisa.

\section{Religiosidade e uso do álcool}

A religiosidade ou espiritualidade, geralmente, é enfatizada como um elemento importante na recuperação da adição, ou mesmo como um fator protetor fundamental contra o abuso de álcool/drogas; varia de acordo com a importância dada ao papel da religiosidade na vida das pessoas (Soldera, Dalgalarrondo \& Correa Filho, 2004). A religiosidade foi verificada neste estudo a partir da afiliação religiosa (católica, evangélica, espírita ou outras) e o quanto a pessoa se considera religiosa, avaliada em uma escala de quatro pontos variando de "nada religioso" a "muito religioso".

Os dados indicam, em conformidade com a literatura citada, que a religião/religiosidade exerce influência sobre o consumo de álcool. Os/as adolescentes que nunca beberam declararam-se, em sua maioria, evangélicos (42\%) e religiosos ou muito religiosos $(58,2 \%)$. Isso pode ser explicado pelo discurso religioso contrário ao consumo de bebidas alcoólicas, bem como pela suposta maior observância de tais preceitos por parte das pessoas mais religiosas. Em oposição, a maior parte dos/as participantes que fizeram uso do álcool identificaram-se como pouco ou nada religiosos, atingindo um percentual de $51 \%$, embora $52 \%$ tenha se afirmado católico, ressaltando-se que $20 \%$ revelaram não ter religião.

É possível observar diferenças significativas na postura perante o consumo de álcool, de acordo com a religião professada. Nusbaumer (1981), analisando os dados obtidos pelo National Opinion Research Center, observou que algumas vertentes protestantes históricas, como a batista e a metodista, tinham maior tendência à abstinência alcoólica 
que os católicos, os luteranos, os presbiterianos e os episcopais. Esse dado foi muito semelhante ao encontrado posteriormente por Engs Hanson, Gliksman e Smythe (1990), que verificaram que os católicos e os protestantes liberais apresentavam mais problemas relacionados com o consumo de álcool do que os protestantes conservadores (batistas e metodistas). Em linhas gerais, os diversos estudos colocam os católicos como o grupo religioso com o maior índice de consumo de álcool, com taxas muito parecidas às das pessoas sem religião. No entanto, é preciso considerar que, no Brasil, grande parte da população autodenomina-se católico, mesmo quando não pertence a nenhum grupo religioso ou é simpatizante de outros.

\section{Trabalho e uso de álcool}

0 trabalho remunerado foi outro fator que apareceu numa frequência diferente nos dois grupos, estando ele mais presente entre os estudantes que beberam, com um percentual de $16 \%$, enquanto que entre os que nunca experimentaram álcool foi de $10 \%$. Isso pode estar relacionado com a maior liberdade e independência que o trabalho proporciona, ocasionando uma menor sujeição de limites impostos pelos pais para com os adolescentes, relação esta já evidenciada na literatura (Amaral, 2008; Soldera, Dalgalarrondo \& Correa Filho, 2004). Segundo Wu, Schlenger e Galvin (2003), são várias as interpretações acerca da associação do consumo de álcool com o trabalho entre adolescentes, podendo estar associado à apresentação e contato com o álcool por pessoas mais velhas no ambiente de trabalho, o baixo compromisso com a escola e a transição precoce para os papéis de adulto. A estes Soldera, Dalgalarrondo e Correa Filho (2004) acrescentam mais três fatores: o estresse pelas responsabilidades decorrentes de uma função adulta assumida precocemente, a disponibilidade financeira proveniente da remuneração e a socialização ocorrida no ambiente de trabalho, levando, por exemplo, às saídas para beber no fim do expediente.

\section{Satisfação com a vida e uso de álcool}

Observou-se ainda que os estudantes que já utilizaram álcool apresentam uma satisfação com a vida menor do que os que nunca beberam, resultado estatisticamente significativo $(p=0,001)$. De acordo com o relatório do CEBRID (Carlini et al., 2006), os jovens precisam sentir que são bons em alguma atividade, sendo que esse destaque representará sua identidade e sua função dentro do grupo. 0 adolescente que não consegue se destacar nos esportes, estudos e relacionamentos sociais, dentre outras ações, pode buscar no álcool sua identificação, cujos efeitos podem proporcionar, de forma imediata, uma melhora desses sintomas, sendo uma tentativa de "automedicação". Quanto mais impulsivo e menos tolerante à frustração for o adolescente, maior será o risco de usar drogas. Segundo estudo desenvolvido com adolescentes dependentes, aqueles que apresentavam sintomas depressivos evoluíam mais rápido da experimentação para o uso regular e também consumiam drogas mais fortes, como a cocaína, em alguns casos, sem ter usado substâncias mais "leves" anteriormente, como a maconha.

\section{Gênero e uso de álcool}

Na análise por gênero, observaram-se diferenças estatisticamente significativas entre as variáveis uso de álcool na vida $(\mathrm{p}=0,03)$, idade de experimentação $(p=0,001)$, frequência do beber no último mês $(p=0,003)$, motivação para a beber $(p=0,001)$. Não houve diferença significativa quanto às consequências do uso do álcool. Dentre aqueles que já ingeriram bebida alcoólica, 44\% são homens e 56\% são mulheres, resultado de relevância uma vez que demonstram que mais mulheres estão experimentando álcool, contrariando a literatura. Em outros estudos realizados (Silva et al., 2006), os adolescentes masculinos apresentaram maior consumo de bebidas alcoólicas.

Os resultados são também indicadores de um incremento do consumo de bebida alcoólica entre o gênero feminino. As adolescentes também foram maioria entre os que beberam no mês anterior à pesquisa (52\%), resultado diverso do que foi obtido no estudo de Horta, Pinheiro, Morales e Strey (2007), no qual o gênero masculino foi predominante. Os dados indicam que as adolescentes, além de experimentarem mais bebida alcoólica que o gênero masculino, também estão na frente quando se trata de consumo regular de álcool. Na presente pesquisa, quando se analisou a frequência do beber nos últimos 30 dias, as estudantes foram maioria no intervalo de 1 a 5 vezes (52\%), porém, os garotos ficaram na frente no intervalo de 6 ou mais vezes, com um percentual de $58 \%$, indicando 
que, na amostra deste estudo, um número maior de meninas bebem, mas para o sexo masculino, há uma maior frequência no beber. A iniciação ao consumo se dá um pouco mais cedo entre os homens, cuja média foi de 13 anos ( $D P=2,2$ ), enquanto que para as mulheres foi de 14 (DP = 1,9). Embora a idade de iniciação seja maior para as mulheres, não se afasta muito da dos homens, sendo ambas bastante precoces.

Embora os homens ainda sejam os maiores consumidores de álcool, observa-se que cada vez mais as mulheres também estão consumindo bebidas alcoólicas com maior frequência e quantidade. Dados confirmam que, mesmo que o consumo de álcool seja realmente menor entre as mulheres, seu impacto pode ser maior que entre os homens, avaliado por meio do relato de problemas associados ao álcool, sendo relatadas, inclusive, mais tentativas de suicídio (Bongers, Van Oers, Vande Goor \& Garretsen, 1997). Comparando o consumo de álcool por gênero em estudo brasileiro (Kerr-Corrêa et al., 2008), os resultados mostraram que mulheres e homens tiveram padrões similares de consumo de álcool. No entanto, no grupo feminino, foram encontrados mais consumidores leves, moderados e problemáticos; no grupo masculino prevaleceram bebedores pesados, mas com menor incidência de bebedores problemáticos.

\section{Motivação/Consequências do uso do álcool}

A literatura acerca do tema tem associado o comportamento de beber a expectativas positivas quanto aos efeitos positivos do uso do álcool por parte dos usuários (Amaral, 2008). Dentre os motivadores levantados neste estudo, as mulheres foram mais frequentes que os homens no fator "faz esquecer as coisas ruins" e "aumenta a simpatia, alegria e animação", enquanto, nos demais, a maior percentagem foi de homens, com destaque para a ajuda nas relações sexuais. É, socialmente, exigida dos homens uma iniciação sexual precoce e uma vida sexual ativa como símbolos de virilidade e masculinidade, bem como há, por parte do grupo de amigos, uma pressão para se adequar a esse modelo. Assim, o álcool pode funcionar para esses adolescentes como facilitadores nesse processo, a partir da desinibição e aumento das relações sociais, fazendo-o sentir-se aceito e bem visto por seus pares. Além disso, a bebida geralmente está presente, principalmente nas festas com os amigos, e o beber em si é visto como prova de maturidade e masculinidade.
Em contrapartida, os fatores nos quais a maioria de respondentes foi do gênero feminino, estão relacionados com uma figura mais recatada, característica da mulher na sociedade. Nota-se ainda que os fatores são opostos entre os gêneros, ou seja, os mais citados pelo gênero feminino são os menos citados pelo gênero masculino e a relação inversa também é verdadeira. Sendo assim, pode-se inferir que as expectativas masculinas são diferentes das que as mulheres possuem com relação ao consumo do álcool, o que pode repercutir na forma de beber de ambos os gêneros e nas consequências advindas dessa prática. Esses resultados podem ser observados na Tabela 2.

\section{Tabela 2 - Fatores motivadores do comportamento de beber}

\begin{tabular}{|lll}
\hline Motivadores do uso do álcool & Feminino & Masculino \\
\hline Faz esquecer coisas ruins & $\mathbf{6 0 \%}$ & $40 \%$ \\
\hline $\begin{array}{l}\text { Aumenta a simpatia, alegria e } \\
\text { animação }\end{array}$ & $\mathbf{5 3 \%}$ & $47 \%$ \\
\hline $\begin{array}{l}\text { Aceitação ou influência do } \\
\text { grupo de amigos }\end{array}$ & $43 \%$ & $57 \%$ \\
\hline $\begin{array}{l}\text { Faz tudo parecer mais fácil } \\
\text { Desinibe e ajuda no relaciona- } \\
\text { mento social }\end{array}$ & $39 \%$ & $61 \%$ \\
\hline \begin{tabular}{l} 
Ajuda nas relações sexuais \\
\hline
\end{tabular} & $12,5 \%$ & $\mathbf{8 7 , 5 \%}$ \\
\hline
\end{tabular}

*Resultados significativos com $\mathrm{p}=0,00$

Fonte: Dados da pesquisa.

O uso do álcool tem sido associado na literatura com diversos comportamentos de risco, como práticas sexuais de risco (Amaral, 2008) e comportamentos de violência (Shepherd, Sutherland \& Newcombe, 2006), onde o álcool aparece como fator facilitador. Na presente pesquisa, esses comportamentos foram revelados por $3 \%$ e $2 \%$ dos participantes, respectivamente, dado que merece atenção visto o $\mathrm{N}$ da amostra. Um número maior de adolescentes (12\%) afirmou já ter feito algo que não faria se não estivesse sobre o efeito do álcool. Verificando as consequências do consumo de álcool segundo o gênero, observa-se uma diferença considerável, principalmente no que diz respeito às brigas e às práticas sexuais, em favor do gênero masculino (Tabela 3), embora não seja estatisticamente significativa. 
Tabela 3 - Consequências do uso do álcool

\begin{tabular}{lll}
\hline $\begin{array}{l}\text { Consequências da ingestão de } \\
\text { álcool }\end{array}$ & Feminino & Masculino \\
\hline Nada & $58,5 \%$ & $41,5 \%$ \\
\hline Brigas & $12,5 \%$ & $87,5 \%$ \\
\hline Fiz algo que não faria & $56 \%$ & $44 \%$ \\
\hline Ressaca & $52 \%$ & $48 \%$ \\
\hline Prática sexual & $32 \%$ & $68 \%$ \\
\hline
\end{tabular}

*Resultados estatisticamente não significativos

Fonte: Dados da pesquisa.

Provavelmente tais diferenças estão ligadas às construções de gênero, em que o homem é, não apenas estimulado, mas cobrado a ter uma vida sexual ativa; e é tido como forte, destemido, naturalizando-se, assim, a agressividade masculina. Desse modo, o efeito do álcool pode atuar como propiciador dessas práticas, já esperadas pela sociedade e incutidas no imaginário dos indivíduos. Robbins e Martin (1993) atestam que comportamentos de homens e mulheres alcoolizados diferem. Os homens teriam ações mais direcionadas ao externo, enquanto as mulheres tendem a comportamentos retraídos, internalizar as emoções e não se expor muito. Apesar de em menor proporção, os dados relativos ao gênero feminino devem ser levados em consideração, visto que podem indicar a difusão entre os gêneros de comportamentos considerados caracteristicamente masculinos, além de se constituírem fatores de vulnerabilidade.

Sabe-se também da existência de uma associação entre as diversas drogas. Um estudo brasileiro (Ferigolo et al., 2004) verificou que a probabilidade de um usuário de álcool usar experimentalmente tabaco, maconha, cocaína ou solventes foi 8 a 11 vezes maior do que entre não usuários. A teoria da progressão do uso de drogas afirma que os adolescentes envolvem-se primeiramente com o álcool, passam em seguida para a maconha e progridem, posteriormente para drogas mais pesadas, como a cocaína.

\section{Considerações finais}

Os resultados deste estudo destacam que um elevado índice dos adolescentes investigados já fez uso de álcool, com preponderância do gênero feminino, confirmando a inversão do perfil socialmente definido que coloca os homens como os consumidores. Este fato torna-se relevante na medida em que se acredita que a ação de drogas como o álcool, capaz de causar desinibição e aumento do desejo sexual, deixe os indivíduos (em especial os adolescentes) mais propensos a práticas sexuais de risco, além de outros agravos.

O consumo de álcool, assim como diversos outros comportamentos parece estar associado a questões de gênero e outras variáveis. 0 gênero dos participantes esteve associado neste estudo a distintos padrões de contato com essa substância, o que pode estar relacionado com condições históricas e sociais determinadas pelo modo como se constroem os conceitos de masculino e feminino.

Os resultados aqui descritos podem fornecer indicativos de um movimento que levaria ao estabelecimento de padrões de consumo de álcool maior para mulheres do que para homens em gerações futuras, o que se constitui numa preocupação imediata não apenas do ponto de vista da saúde pública, mas principalmente na ótica da saúde da mulher. Tal movimento pode estar associado à emancipação das mulheres e à crescente conquista de espaço social por parte deste segmento da população, bem como à consequente mudança do seu papel na sociedade. Como comenta Horta et al. (2007), seria lamentável que as lutas pela democratização, emancipação das mulheres não viessem acompanhadas de uma reflexão crítica a respeito do que as coloca em risco.

Os altos índices de consumo de álcool pelas adolescentes encontrados neste estudo, despertam uma preocupação quanto ao futuro dessas jovens visto que, quanto mais cedo se dá a iniciação ao uso de álcool, maior a vulnerabilidade para o desenvolvimento de abuso e dependência dessa substância e o uso concomitante de outras drogas. Sendo assim, se não receber a atenção necessária e as devidas providências não forem tomadas, esse quadro pode ser precedente de um mais grave - o alcoolismo -, podendo atingir proporções desastrosas para a população feminina.

O panorama apresentado parece antever sérios problemas a serem enfrentados no futuro no que diz respeito à saúde das populações, visto que o consumo de álcool pode resultar em ocorrências letais, mas de lento impacto em relação a mudanças de padrões de consumo. As políticas públicas, 
sejam elas setoriais ou institucionais, são apontadas, então, como uma estratégia capaz de desestimular, conter ou contribuir para a reversão desses comportamentos (Horta et al., 2007). Dada a complexidade da temática, faz-se necessária a inclusão e o envolvimento nos debates de movimentos sociais e setores diversos da sociedade civil e mídia, além de gestores públicos.

Tendo em vista que a amostra deste estudo se restringe a adolescentes estudantes, que frequentam a escola, residentes na capital, os perfis de consumo aqui encontrados não podem ser aplicados à população adolescente de forma geral. Nesse sentido, sugere-se a realização de outros estudos mais abrangentes que possam investigar adolescentes com outras características a fim de constatar se os padrões de comportamentos em relação ao álcool permanecem os mesmos.

\section{Agradecimentos}

Os autores agradecem ao CNPq pelo financiamento do estudo.

\section{Referências}

Amaral, A. C. G. (2008). 0 uso do álcool e a vulnerabilidade à Aids: Estudo com adolescentes gaúchos e paraibanos. Dissertação de Mestrado. Pós-Graduação em Psicologia Social, Universidade Federal da Paraíba, João Pessoa.

Araújo, L. B., \& Gomes, W. B. (1998). A adolescência e as expectativas em relação aos efeitos do álcool. Psicologia, Reflexão e Crítica, 11(1), 5-33.

Bongers, I. M., Van Oers, H. A., Van De Goor, I. A., \& Garretsen, H. F. (1997). Alcohol use and problem rinking: Prevalences in the general Rotterdam population. Substance Use \& Misuse, 32(11), 1491-512.

Cardim, M. S., \& Azevedo, B. A. (1995). Antecedentes familiares na determinação da gravidade do alcoolismo. Informe Psiquiatria, 14(1), 5-12.

Carlini, E. A., Carlini-Cotrim, B., Silva-Filho, A. R., \& Barbosa, M. T. S. (1990). Levantamento Nacional sobre o uso de psicotrópicos em estudantes de $1^{\circ}$ e $2^{\underline{o}}$ Graus. CEBRID - Centro Brasileiro de Informações sobre Drogas Psicotrópicas, 20(2) 241-257.
Carlini, E. A., Galduróz, J. C. F., Noto, A. R., \& Nappo, S. A. (Org.). (2002). I levantamento domiciliar sobre o uso de drogas psicotrópicas no Brasil. São Paulo: Centro Brasileiro de Informações sobre Drogas Psicotrópicas.

Carlini, E. A., Galduróz, J. C. F., Noto, A. R., Fonseca, A. M., Carlini, C. M., Oliveira, L. G., et al. (2006). II Levantamento Domiciliar sobre o Uso de Drogas Psicotrópicas no Brasil: Estudo envolvendo as 108 maiores cidades do País - 2005. Brasília: Secretaria Nacional Antidrogas.

Costa-e-Silva, V. L., \& Koifman, S. (1998). Smoking in Latin America: A major public health problem. Cadernos de Saúde Pública, 14(3), 99-108.

De Bem, M. F. L. (2003). Estilo de Vida e Comportamentos de risco de estudantes trabalhadores do ensino médio de Santa Catarina. Tese de Doutorado em Engenharia de Produção - Ergonomia, Universidade Federal de Santa Catarina, Florianópolis.

Duque-Arrazola, L. S. (1997). 0 cotidiano sexuado de meninos e meninas em situação de pobreza. In F. R. Madeira (Org.). Quem mandou nascer mulher? (pp. 343-402) Rio de Janeiro: Record; Rosa dos Tempos.

El-Guebaly, N. (2006). Don't drink and drive: The successful message of mothers Against Drunk Driving (MADD). World Psychiatry, 4(1), 35-36.

Engs, R. C., Hanson, D. J., Gliksman, L., \& Smythe, C. (1990). Influence of religion and culture on drinking behaviors: A test of hypotheses between Canada and USA. British Journal of Addiction, 85(11), 1475-1482.

Farias Jr, J. C. (2002). Estilo de vida de escolares do ensino médio no município de Florianópolis, Santa Catarina, Brasil. Dissertação de Mestrado em Educação Física, Universidade Federal de Santa Catarina, Florianópolis.

Ferigolo, M., Barbosa, F. S., Arbo, E., Malysz, A. S. Stein, A. T., \& Barros, H. M. T. (2004). Prevalência do Consumo de Drogas na FEBEM, Porto Alegre. Rev Bras Psiquiatr, 26(1), 9-15.

Galduróz, J. C. F, Noto, A. R., Fonseca, A. M., \& Carlini, E. A. (2004). V Levantamento Nacional sobre o Consumo de Drogas Psicotrópicas entre Estudantes do Ensino Fundamental e Médio da Rede Pública de Ensino nas 27 Capitais Brasileiras - 2004. São Paulo: CEBRID. 
Grimberg, M. (1999). Sexualidad y relaciones de género: Una aproximación a la problemática de la prevención del VIH/sida en sectores populares de la ciudad de Buenos Aires. Cuadernos Médico Sociales, 75, 65-76.

Hennigen, I., \& Guareschi, N. M. F. (2002). A paternidade na contemporaneidade: Um estudo de mídia sob a perspectiva dos estudos culturais. Psicologia e Sociedade, 14(1), 44-68.

Horta, R. L., Horta, B. L., Pinheiro, R. T., Morales, B., \& Strey, M. N. (2007). Tabaco, álcool e outras drogas entre adolescentes em Pelotas, Rio Grande do Sul, Brasil: Uma perspectiva de gênero. Cadernos de Saúde Pública, 23(4), 775-783.

Keller, M. (1980). Concepções sobre o alcoolismo. Revista ABP APAL, 2(2), 93-100.

Kerr-Corrêa, F., Tucci, A. M., Hegedus, A. M., Trinca, L. A., Oliveira, J. B., Floripes, T. M. F., et al. (2008). Diferenças nos padrões de consumo de álcool entre homens e mulheres em duas comunidades brasileiras distintas. Revista Brasileira de Psiquiatria, 30(3), 35-42.

Korin, D. (2001). Novas perspectivas de gênero em saúde. Adolescência Latinoamericana, 2(2), 67-79.

Kroeff, L. R., Sotero, S. M., Schmidt, M. I., Duncan, B. B., Favaretto, A. L. F., \& Nucci, L. B. (2004). Fatores associados ao fumo em gestantes avaliadas em cidades brasileiras. Revista de Saúde Pública, 38(2), 261-267.

Larossa, E. (1989). Fatherhood and social change. Men's Studies Review, 6(2), 1-9.

Michaud, P. A. (2001). Pesquisa multicultural sobre saúde do adolescente: Uma perspectiva em saúde pública. Adolescência Latinoamericana, 2(3), 152-158.

Nusbaumer, M. R. (1981). Religious affiliation and abstinence: A fifteen-year change. Journal of Studies on Alcohol, 42(1), 127-131.

Pechamsky, F. (2001). Modelo teórico de exposição a risco para transmissão do vírus HIV em usuários de drogas. Rev Bras Psiquiatr, 23(1), 41-47.

Pinsky, I., \& Silva, M. T. (1999). A frequency and content analysis of alcohol advertising on Brazilian television. Journal of Studies on Alcohol, 60(3), 394-399.
Robbins, C. A., \& Martin, S. S. (1993). Gender, styles of deviance, and drinking problems. Journal of Health and Social Behavior, 34(4), 302-21.

Schenker, M., \& Minayo, M. C. S. (2003). A implicação da família no uso abusivo de drogas: Uma revisão crítica. Ciência e saúde coletiva, 8(1), 229-306.

Scivoletto, S. (1997). Tratamento psiquiátrico ambulatorial de adolescentes usuários de drogas - características sociodemográficas, a progressão do consumo de substâncias psicoativas e fatores preditivos de aderência e evolução no tratamento. Tese de Doutorado em Medicina, Universidade de São Paulo, São Paulo.

Shepherd, J. P., Sutherland, I., \& Newcombe, R. G. (2006). Relations between alcohol, violence and victimization in adolescence. Journal of Adolescence, 29(4), 539-553.

Soldera, M., Dalgalarrondo, P., \& Correa Filho, H. (2004). Uso de drogas psicotrópicas por estudantes: Prevalência e fatores sociais associados. Revista de Saúde Pública, 38(12), 277-283.

Souza, D. P. O., Areco, K. N., \& Silveira Filho, D. X. (2005). Álcool e alcoolismo entre adolescentes da rede estadual de ensino de Cuiabá, Mato Grosso. Revista de Saúde Pública, 39(4), 585-592.

Traverso-Yépez, M. A., Pinheiro, V. S. (2005). Socialização de gênero e adolescência. Estudos Feministas, 13(1), 147-162.

U.S. Departament of Health and Human Services. Center for Disease Control and Prevention. Youth Risk Behavior Surveillance System (BRFSS). (1999). Recuperado em: 15 mar. 2008, em, http: www.cdc.gov

Wang, M., Jablonski, B., \& Magalhaes, A. S. (2006). Identidades masculinas: Limites e probabilidades. Psicologia em Revista, 12(19), 54-65.

Wu, L. T., Schlenger, W. E., \& Galvin, D. M. (2003). The relationship between employment and substance use among students aged 12 to 17 . Journal Adolescent Health, 32(1), 5-15. 
